\title{
Utilization of multiparametric prostate magnetic resonance imaging in clinical practice and focal therapy: report from a Delphi consensus project
}

\author{
M. J. Scheltema ${ }^{1} \cdot$ K. J. Tay ${ }^{3}$ A. W. Postema ${ }^{1}$ D. M. de Bruin ${ }^{1,2} \cdot$ J. Feller ${ }^{5}$ - J. J. Futterer ${ }^{6}$ A. K. George ${ }^{7}$. \\ R. T. Gupta ${ }^{4}$ - F. Kahmann ${ }^{8}$ - C. Kastner ${ }^{9}$ - M. P. Laguna ${ }^{1}$ - S. Natarajan ${ }^{10}$ - S. Rais-Bahrami ${ }^{11}$ \\ A. R. Rastinehad ${ }^{12,13}$ - T. M. de Reijke ${ }^{1}$ G. Salomon ${ }^{15}$ N. Stone S2,14 $^{12}$ R. van Velthoven ${ }^{16} \cdot$ R. Villani $^{17}$. \\ A. Villers $^{18}$ • J. Walz ${ }^{19}$ - T. J. Polascik ${ }^{3}$ · J. J. M. C. H. de la Rosette ${ }^{1}$
}

Received: 1 August 2016 / Accepted: 6 September 2016 / Published online: 16 September 2016

(c) The Author(s) 2016. This article is published with open access at Springerlink.com

\begin{abstract}
Purpose To codify the use of multiparametric magnetic resonance imaging (mpMRI) for the interrogation of prostate neoplasia $(\mathrm{PCa})$ in clinical practice and focal therapy (FT).

Methods An international collaborative consensus project was undertaken using the Delphi method among experts in the field of PCa. An online questionnaire was presented in three consecutive rounds and modified each round based on the comments provided by the experts. Subsequently,
\end{abstract}

Electronic supplementary material The online version of this article (doi:10.1007/s00345-016-1932-1) contains supplementary material, which is available to authorized users.

\section{J. Scheltema}

m.j.scheltema@amc.uva.nl

1 Department of Urology, Academic Medical Center, Amsterdam, The Netherlands

2 Department of Biomedical Engineering and Physics, Academic Medical Center, Amsterdam, The Netherlands

3 Department of Surgery, Duke University Medical Center, Durham, NC, USA

4 Department of Radiology, Duke University Medical Center, Durham, NC, USA

5 Desert Medical Imaging, Indian Wells, CA, USA

6 Department of Radiology, Radboud University Nijmegen Medical Centre, Nijmegen, The Netherlands

7 Urologic Oncology Branch, National Cancer Institute, National Institutes of Health, Bethesda, MD, USA

8 Urologische Praxis Dr. Henkel and Dr. Kahmann, Berlin, Germany

9 CamPARI Prostate Cancer Clinic, Cambridge University Hospitals Trust, Cambridge, UK a face-to-face meeting was held to discuss and finalize the consensus results.

Results mpMRI should be performed in patients with prior negative biopsies if clinical suspicion remains, but not instead of the PSA test, nor as a stand-alone diagnostic tool or mpMRI-targeted biopsies only. It is not recommended to use a 1.5 Tesla MRI scanner without an endorectal or pelvic phased-array coil. mpMRI should be performed following standard biopsy-based PCa diagnosis in both the planning and follow-up of FT. If a lesion is seen, MRI-TRUS fusion biopsies should be performed for FT planning. Systematic biopsies are still required for FT planning in biopsynaïve patients and for patients with residual PCa after FT.

10 Department of Urology, Surgery and Bioengineering, University of California, Los Angeles, CA, USA

11 Department of Urology and Radiology, The University of Alabama at Birmingham, Birmingham, AL, USA

12 Department of Urology, Icahn School of Medicine at Mount Sinai, New York, NY, USA

13 Department of Radiology, Icahn School of Medicine at Mount Sinai, New York, NY, USA

14 Department of Radiation Oncology, Icahn School of Medicine at Mount Sinai, New York, NY, USA

15 Martini-Clinic Prostate Cancer Center, University Hospital Hamburg-Eppendorf, Hamburg, Germany

16 Department of Urology, Institut Jules Bordet, Brussels, Belgium

17 Department of Radiology, North Shore University Hospital, Northwell Health, NY, USA

18 Department of Urology, Lille University Medical Center, Lille, France

19 Department of Urology, Institut Paoli-Calmettes Cancer Centre, Marseille, France 
Standard repeat biopsies should be taken during the followup of FT. The final decision to perform FT should be based on histopathology. However, these consensus statements may differ for expert centers versus non-expert centers.

Conclusions The mpMRI is an important tool for characterizing and targeting $\mathrm{PCa}$ in clinical practice and FT. Standardization of acquisition and reading should be the main priority to guarantee consistent mpMRI quality throughout the urological community.

Keywords Focal therapy · Prostate cancer · Consensus · Magnetic resonance imaging $\cdot$ Delphi

\section{Introduction}

Recent technological advancements in multiparametric magnetic resonance imaging (mpMRI) have resulted in improved detection of clinically significant prostate cancer (PCa) and are increasingly used in urological practice and for focal therapy (FT). MpMRI most commonly includes T1-2-weighted imaging, dynamic contrast-enhanced (DCE) and diffusion-weighted imaging (DWI), providing clinicians with meaningful information regarding lesion volume, morphology, location and disease extent. Dynamic threedimensional lesion characterization and risk assessment with mpMRI is key for adequate patient selection and treatment planning for FT. Clinical guidelines for standardized reporting and acquisition (e.g., PI-RADS v2 [1] or Likert scale) of prostate mpMRI are nowadays advised for both research and clinical practice. When comparing mpMRI and pathology following radical prostatectomy (sliced by use of a customized 3D mold), the positive predictive value (PPV) for the detection of $\mathrm{PCa}$ in the peripheral zone, central zone and overall prostate was 98,100 and $98 \%$, respectively, whereas the negative predictive value (NPV) was $90 \%$ for all mpMRI sequences [2]. In another series, the positive and negative predictive values were 86 and $85 \%$ for lesions $>0.2 \mathrm{~mL}$ and 77 and $95 \%$ for lesions $>0.5 \mathrm{~mL}$ [3]. Noteworthy, most excellent results on mpMRI PCa detection are published by expert centers where the quality of the mpMRI is assured by standardized acquisition, interpretation and image-pathology feedback. Hence, results may not be reflective to general urological practice (for an overview table on mpMRI PCa detection see $[4,5])$. To illustrate, a recent systematic review of available literature on the detection of significant PCa by mpMRI showed that the NPV ranged from 63 to $98 \%$ [6]; however, the majority of the included studies used prostate biopsies for histopathological validation.

Of interest for FT of the index lesion, the PPV of mpMRI was reported to be $82.6 \%$ [7], whereas $80 \%$ of all index tumors and $72 \%$ of Gleason $\geq 7$ tumors on whole mount pathology were identified by mpMRI [8]. Moreover,
mpMRI-transrectal ultrasound (TRUS) fusion (cognitive and system-based) targeted biopsies (TB) has shown to decrease the detection of clinically insignificant $\mathrm{PCa}$ and increase de detection rate of clinically significant $\mathrm{PCa}$ with an absolute difference of $6.8 \%$ between mpMRI-TRUS fusion TB versus TRUS-guided biopsies [9]. However, not all studies reached a statistically significant difference [9-15] (for systematic review see [9, 15]). Expert panels recommend to perform repeated mpMRI during surveillance following FT [4]. MpMRI-TRUS fusion TB has been shown to increase the detection of clinically significant $\mathrm{PCa}$ in the follow-up during active surveillance or during confirmatory biopsies of patients with previous negative TRUS-guided biopsies [16-18]. With increasing evidence, the position of mpMRI and MR-targeted biopsies to the accepted standard of PCa diagnostics needs to be re-evaluated. Therefore, an international multidisciplinary consensus project was initiated using the Delphi method, aiming to define the use of mpMRI and MR-targeted biopsies in clinical practice and focal therapy of PCa.

\section{Methods}

\section{The Delphi method}

This consensus project was executed following the Delphi method [19]. In short, a systematic literature search was conducted after which experts were selected and invited to participate. An online survey using online questionnaire software (www.SurveyMonkey.com) was constructed and presented to participants in three consecutive rounds. Only experts that completed all previous rounds were reinvited to participate in the subsequent round. After each round, the questionnaire was modified based on the comments provided by experts and aggregated results of the previous round were anonymously presented to allow the experts to re-evaluate their answers without peer-pressure. Subsequently, a face-to-face meeting was held to discuss the results of the online survey.

A systematic literature search of the PubMed database was performed on February 18, 2016 (see Fig. 1.), with filters: English language, full-text availability and human studies. Articles involving salvage therapy, reviews or small pilot-trials $(n \leq 10)$ were excluded. After reviewing the literature, 166 experts were invited to participate based on authorship or peer-recommendation. The online consensus process was performed between March 21 and May 27, 2016. The percentage of unanimity that should be reached to reach consensus was set at $80 \%$. A face-to-face panel meeting was organized on June 23, 2016, during the 9th International Symposium on Focal Therapy and Imaging in Prostate and Kidney Cancer (www.focaltherapy.org) and 


\section{Flow Chart Search}

((( (focal [Title/abstract] OR focal therapy [Title/abstract] OR focal treatment [Title/abstract] OR localized treatment [Title/abstract]

OR ablation [Title/abstract] OR ablative therapy [Title/abstract]) AND (multiparametric magnectic resonance imaging

[Title/abstract] OR mpMRI OR multiparametric MRI [Title/abstract] OR magnectic resonance imaging [Title/abstract] OR MRI

[Title/abstract])) AND (carcinoma [Title/abstract] OR cancer [Title/abstract] OR neoplasm [Title/abstract] OR neoplasms

[Title/abstract] OR neoplasma [Title/abstract] OR neoplasmata [Title/abstract] OR malignancy [Title/abstract] OR malignant

[Title/abstract] OR adenocarcinoma [Title/abstract] OR adenocarcinom [Title/abstract] OR tumor [Title/abstract] OR tumour

[Title/abstract] OR cancers [Title/abstract])) AND (prostate [Title/abstract] OR prostatic [Title/abstract] OR prostatae

[Title/abstract]))

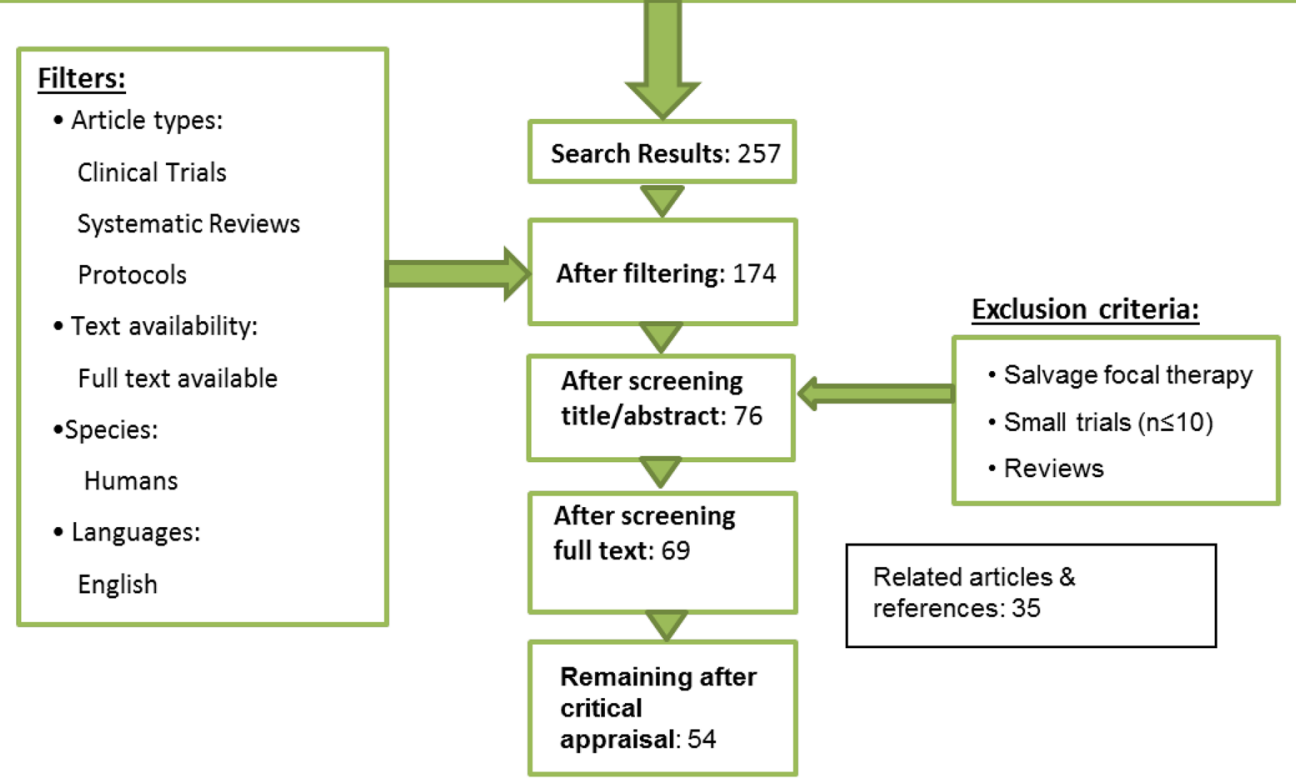

Fig. 1 Systematic literature search

was attended by 17 participants (94\% completed all online rounds). Panelists discussed inconclusive results from the questionnaire in detail, as by definition results with online confirmed consensus cannot be changed. For an overview of the final results of the online questionnaire and face-toface panel outcomes, see in ESM 1.

\section{Results}

\section{Consensus process and background of participants}

Ninety experts (90/166) accepted the invitation, and the response rate for the questionnaire was $100 \%$ (90/90), $94 \%$ (85/90), $88 \%$ (79/90) for rounds 1, 2, and 3, respectively, and $87 \%$ (78/90) completed all three rounds. In this group of experts $72 \%$ were urologists, $16 \%$ radiologists, $3 \%$ pathologists, $3 \%$ radiation oncologists and $6 \%$ scientists. Average clinical experience in the field of PCa was 18.9 years (SD 9.6), 1567 years in total, and include experience with the following focal ablative modalities (primary and/or salvage); cryosurgery ( $n=11)$, high-intensity focused ultrasound $(n=22)$, irreversible electroporation $(n=11)$, laser therapy $(n=5)$, vascular-targeted photodynamic therapy $(n=8)$, other $(n=4)$ and not specified/no FT $(n=29)$. Some participants had experience with more than one ablative modality for FT. A MRI-TRUS fusion system is used by $83 \%(64 / 77)$ of clinicians in clinical practice to guide prostate biopsies or focal treatment procedures. A standardized mpMRI protocol (85\% PI-RADS, $7 \%$ Likert, $2 \%$ both PI-RADS/Likert, $2 \%$ other) is used by $96 \%(79 / 82)$ of clinicians. In conclusion, this group may provide a valid expert opinion due to the aforementioned experience of the participants with the utility of mpMRI and targeted biopsies in both clinical practice and focal therapy of PCa. For a list of all participants of the online survey and panel meeting, see in ESM 2.

\section{mpMRI in clinical urological practice}

It is not recommended to use a 1.5 Tesla MRI scanner without an endorectal coil (ERC) or pelvic phased-array coil (81 \% of 
whole group against, $92 \%$ of radiologist against) and $83 \%$ of the radiologists were also against the use of a $1.5 \mathrm{~T}$ with a pelvic phased-array coil only. The panel emphasized that with older 1.5T systems, the use of an ERC is indispensable. When, however, newer generation 1.5T systems are used and/ or if an experienced radiologist optimizes other acquisition parameters, good image quality can be obtained with $1.5 \mathrm{~T}$ scanners without the use of an ERC. This is in line with the recommendations of the PI-RADS Steering Committee [1].

The use of mpMRI in the workup for patients suspected of PCa was codified, and consensus was reached that mpMRI should be performed after the first set of negative TRUS-guided biopsies if clinical suspicion remains ( $90 \%$ ), but not instead of the PSA test $(96 \%)$ nor as stand-alone diagnostic tool $(95 \%)$ or TB only (76\% with panel agreement). There was no agreement on whether or not mpMRI should be performed in the workup for all patients with suspicion of $\mathrm{PCa}$, in combination with standard (10-12 core) prostate biopsies and mpMRI-TRUS fusion TB (if lesion is seen). The panel stated that only in expert centers, where the quality is assured and their own results are monitored, mpMRI may be performed in all patients suspected of PCa since it could increase the detection of clinically significant $\mathrm{PCa}$, reduce the need for repeat biopsies and may avoid prostate biopsies in patients with a negative mpMRI [20]. Therefore, it could be economically justifiable, however, adequate cost-benefit studies per health care system are lacking and should be performed.

The experts were divided on whether the current NPV of mpMRI is acceptable in clinical practice to rule out significant $\mathrm{PCa}$ (47\% agree/49\% disagree). The panel refines that it may be applicable for expert centers with a known NPV of $>90 \%$ for clinically significant $\mathrm{PCa}$, but not in community practice. In line, no consensus was reached that, after the first set of negative prostate biopsies, repeat prostate biopsies can be deferred if mpMRI does not show any suspect lesions. However, according to a recent AUASAR consensus statement, deferral of repeat biopsy may be considered in expert centers in case of a negative mpMRI (without a strong clinical suspicion) [21].

If prostate biopsies are performed before mpMRI, the majority recommended the minimum acceptable interval to wait before performing mpMRI to be 6 weeks $(68 \%$ with panel agreement), although it would still impair the imaging quality (97\%). Literature shows that post biopsy hemorrhage was prevalent (57\%) within 6 weeks following prostate biopsies, but did not have a detrimental effect on tumor detection or staging $[22,23]$.

\section{Focal therapy planning}

MpMRI should be performed for FT planning in patients with a TRUS-guided biopsy confirmed PCa (94\%), including T1-2-weighted imaging, DCE and DWI, but without magnetic resonance spectroscopic imaging (MRSI) (83\%). MRI-TRUS fusion (either system or cognitive) is the recommended technique to perform biopsies following mpMRI (93\%) if a radiographic lesion is seen and is deemed targetable. Transperineal template mapping biopsies (TTMB) (58\% in favor with panel agreement) can also be considered, whereas TRUS-guided and in bore MRguided biopsies are the least favorable techniques (58 and $64 \%$ against, respectively). The panel highlighted that all techniques can be considered, depending on availability and experience, but acknowledged MRI-TRUS fusion as the optimal approach. Stand-alone MRI-targeted prostate biopsies are only sufficient for FT planning without systematic (random) biopsies in patients with previous negative TTMB (86 \%), but not in biopsy-naïve patients (93\%) or in patients undergoing repeat biopsies after primary FT for low- to intermediate-risk PCa (81\%). The final decision to perform FT should be based on (targeted) histopathology and should not be based on mpMRI results/PI-RADS score $(86 \%)$ alone. Histopathological confirmation remains crucial since a recent prospective evaluation of the PI-RADS v2 found a relatively low cancer detection rate of 16, 30 and $78 \%$ for PI-RADS score 3, 4 and 5, respectively [24].

Precise Lesion size and extension cannot be adequately assessed based with the current mpMRI quality according to the available scientific data for focal treatment planning $(82 \%)$. In a small cohort $(n=33)$, it was shown that mpMRI underestimated lesion size and boundaries. This underestimation was larger for high suspicion on imaging or higher Gleason score, however, if a simulated safety margin of $9 \mathrm{~mm}$ was applied, all lesions would have been treated completely [25]. The panel agreed that more data are needed to optimize treatment boundaries for FT planning.

\section{mpMRI during and in the follow-up of focal therapy}

MRI-TRUS system fusion is considered to be the best and most practical imaging modality to guide FT procedures (e.g., needle-placement) (86\% agreement). However, the panel remarked that cognitive fusion or other modalities might be acceptable, depending on availability and experience. MpMRI should be part of the follow-up (standardized care) following focal therapy (91\%), excluding MRSI (79\%, with panel agreement). MRI-TRUS fusion biopsies should be performed following mpMRI if a lesion is seen (78 \%, with panel agreement). To illustrate: in 59 patients suspected of recurrent PCa following HIFU, the likelihood of finding $\mathrm{PCa}$ was greater with TB on lesions seen with T2-weighted and DCE-MRI compared with systematic random biopsies [26]. However, mpMRI with MRI-TRUS fusion TB cannot serve as stand-alone follow-up modality 
Table 1 Overview of consensus recommendations

Recommendation

Online or panel agreement

mpMRI should be performed in patients with prior negative biopsies if clinical suspicion remains

Online

mpMRI should not be performed as stand-alone diagnostic tool or with mpMRI-targeted biopsies only

Online

mpMRI should be performed following standard biopsy-based PCa diagnosis in both the planning

Online

and follow-up of FT

MRI-TRUS fusion is the recommended technique to perform biopsies following mpMRI

Online

Systematic biopsies are still required for FT planning in biopsy-naïve patients and patients with residual PCa after FT

Repeat biopsies should be taken during the follow-up of FT

Online

The final decision to perform FT should be based on histopathology and not be based on mpMRI results alone

Only in expert centers, where the quality is assured and own results are monitored, mpMRI may be performed in all patients suspected of $\mathrm{PCa}$

Only in expert centers, deferral of repeat biopsy may be considered in case of a negative mpMRI

Online

Online

Panel

It should be our goal to guarantee high-quality mpMRI throughout the urological community before implementing

it as standard of care

following FT and standard repeat (random) biopsies should be taken ( $78 \%$, with panel agreement). In a paper by Shah et al. on the histological outcomes after focal HIFU and focal cryosurgery, standard repeat biopsies were positive in $25 \%(98 / 391)$ and $22 \%(39 / 175)$ for focal cryosurgery and focal HIFU, respectively [27]. The majority of these positive post-treatment biopsies were clinically insignificant $\mathrm{PCa}$, and whether these lesions would have been identified by mpMRI remains unknown. To the best of our knowledge, there are no large series comparing MR-guided TB with standard systematic repeat biopsies on their ability to detect clinically significant PCa following FT.

\section{Discussion}

This Delphi consensus project represents the opinion of 90 FT experts, experienced with mpMRI and MRI-TRUS fusion (TB), for all recommendations see Table 1. Despite experience, it should still be regarded as expert opinion, considered level 5 evidence, may be biased by personal enthusiasm and is potentially not reflective for communitybased urologists. Nonetheless, these statements can provide clinicians guidance in areas where high-level evidence is sparse and provide a basis for standardization for clinical utilization of mpMRI and/or focal therapy that could result in improved interpretation of reported series.

The main challenge that was repeatedly encountered was the discrepancy regarding the consensus statements that can be made for expert centers versus non-expert centers. For experienced centers, where high-quality mpMRI is obtained by standardized reporting and acquisition, experience by radiologists, and where the own data are known, some excellent results have been published, establishing the mpMRI as a reliable diagnostic tool $[2,3,6]$. In community practice, this process may be less optimal, causing heterogeneous mpMRI quality with decreased sensitivity and specificity, resulting in clinically significant $\mathrm{PCa}$ being missed, understaging and a high number needed to image impairing the potential costeffectiveness [20]. The panel argued that it should be our goal to guarantee high-quality mpMRI throughout the urological community before implementing it as standard of care. Important elements to achieve this are expert training, imaging-pathology feedback and/or certification. Moreover, costbenefit studies must be performed per health care system and the availability of MRI scanners and logistics should improve [28]. Furthermore, it may be inevitable that FT can only be performed in centers where the mpMRI quality is guaranteed, since mpMRI and MRI-TRUS fusion biopsies should be performed in both the planning and follow-up of FT.

This consensus project recommended that mpMRI should be performed in patients with prior negative biopsies if clinical suspicion for $\mathrm{PCa}$ remains, which is stated more firmly than the EAU guideline recommendation that mpMRI may be used to evaluate the need to perform repeat biopsies [28]. Ahmed et al. presented during the 9th International Symposium on Focal Therapy and Imaging in Prostate and Kidney Cancer the first level 1 evidence that using mpMRI as a triage test, in all patients suspected of PCa $(n=576)$, can avoid unnecessary prostate biopsies and detected more clinically significant $\mathrm{PCa}(\geq$ Gleason $4+3$ ) than systematic (random) TRUS-guided biopsies. Based on their results, these authors calculated that in the United Kingdom it was cost-effective to perform mpMRI in all patients suspected of PCa (unpublished data, [20]). This underlines the panel statement that in expert centers mpMRI may be performed in all patients suspected of PCa. The outcomes regarding mpMRI acquisition and reporting are 
following the recommendations by the PI-RADS Steering Committee [1] that shows the potential that the PI-RADS recommendations have to effectuate uniform mpMRI.

Previous consensus meetings differed on the need for systematic biopsies when MRI-TRUS fusion TB were performed in biopsy-naïve patients $[29,30]$. Since mpMRI quality is not constant throughout the field, abandoning systematic biopsies after MRI-TRUS fusion TB for FT planning in biopsy-naïve patients is not recommended by this consensus group. In line, systematic prostate biopsies should be taken during the follow-up after FT.

\section{Conclusions}

In expert centers mpMRI evaluation of the prostate is an established component in $\mathrm{PCa}$ diagnostics and this consensus project aimed to define the use of mpMRI in clinical care, especially in relation to FT. In FT, mpMRI should be used for patient selection, treatment planning or guidance, and post-treatment monitoring. Newly developed MRI-TRUS (system or cognitive) fusion is increasingly being used either during prostate biopsy procedures or during treatment planning or guidance. This consensus project was conducted during the turning point whether or not mpMRI of the prostate should be standard of care in all patients with or suspected of PCa. Standardization of acquisition and reading should be the main priority to guarantee consistent mpMRI quality throughout the field to substantiate the discrepancy between what you want to recommend and what you actually can recommend.

Acknowledgments All participants listed in ESM 2 that greatly helped the conduct of this study by completing the online questionnaires. This consensus project was supported by a Grant of the Cure for Cancer foundation. No commercial funding was received for the conduct of this consensus project.

Author contributions M.J. Scheltema, J. Kay, A.W. Postema, D.M. de Bruin, T.J. Polascik and J.J. De la Rosette were involved in project development, data collection, data analysis, and manuscript writing/ editing; T.M. de Reijke and M.P. Laguna were involved in project development and manuscript writing/editing; and J. Feller, J. Futterer, A.K. George, R.T. Gupta, F. Kahmann, C. Kastner, S. Natarajan, S. Rais-Bahrami, A.R. Rastinehad, G. Salomon, N. Stone, R. van Velthoven, R. Villani, A. Villers and J. Walz were involved in data collection, data analysis and manuscript editing.

\section{Compliance with ethical standards}

This consensus was conducted according to the Declaration of Helsinki and does not involve human subjects.

Conflict of interest The following authors declare no conflict of interest: Scheltema, Tay, Postema, de Bruin, Feller, Futterer, George, Gupta, Kahmann, Kastner, Laguna, Natarajan, Rastinehad, de Reijke, Salomon, van Velthoven, Villani, Villers, Walz, Polascik, De la
Rosette. Stone is owner of 3D Biopsy, Rais-Bahrami is consultant for Philips/Invivo and Gupta is consultant for Invivo.

Open Access This article is distributed under the terms of the Creative Commons Attribution 4.0 International License (http://creativecommons.org/licenses/by/4.0/), which permits unrestricted use, distribution, and reproduction in any medium, provided you give appropriate credit to the original author(s) and the source, provide a link to the Creative Commons license, and indicate if changes were made.

\section{References}

1. Barentsz JO, Weinreb JC, Verma S, Thoeny HC, Tempany CM, Shtern F et al (2016) Synopsis of the PI-RADS v2 guidelines for multiparametric prostate magnetic resonance imaging and recommendations for use. Eur Urol 69(1):41-49. doi:10.1016/j. eururo.2015.08.038

2. Turkbey B, Mani H, Shah V, Rastinehad AR, Bernardo M, Pohida $T$ et al (2011) Multiparametric 3T prostate magnetic resonance imaging to detect cancer: histopathological correlation using prostatectomy specimens processed in customized magnetic resonance imaging based molds. J Urol 186(5):1818-1824. doi:10.1016/j.juro.2011.07.013

3. Villers A, Puech P, Mouton D, Leroy X, Ballereau C, Lemaitre L (2006) Dynamic contrast enhanced, pelvic phased array magnetic resonance imaging of localized prostate cancer for predicting tumor volume: correlation with radical prostatectomy findings. J Urol 176(6):2432-2437

4. Muller BG, Futterer JJ, Gupta RT, Katz A, Kirkham A, Kurhanewicz $J$ et al (2014) The role of magnetic resonance imaging (MRI) in focal therapy for prostate cancer: recommendations from a consensus panel. BJU Int 113(2):218-227

5. Mendez MH, Joh DY, Gupta R, Polascik TJ (2015) Current trends and new frontiers in focal therapy for localized prostate cancer. Curr Urol Rep 16(6):1-11. doi:10.1007/ s11934-015-0513-y

6. Futterer JJ, Briganti A, De Visschere P, Emberton M, Giannarini G, Kirkham A et al (2015) Can clinically significant prostate cancer be detected with multiparametric magnetic resonance imaging? A systematic review of the literature. Eur Urol 68(6):1045-1053

7. Rosenkrantz AB, Deng FM, Kim S, Lim RP, Hindman N, Mussi TC et al (2012) Prostate cancer: multiparametric mri for index lesion localization - a multiple-reader study. Am J Roentgenol 199(4):830-837

8. Le JD, Tan N, Shkolyar E, Lu DY, Kwan L, Marks LS et al (2014) Multifocality and prostate cancer detection by multiparametric magnetic resonance imaging: correlation with wholemount histopathology. Eur Urol [Internet]. European Association of Urology 67(3):1-8. Available from: http://www.ncbi.nlm.nih. gov/pubmed/25257029

9. Valerio M, Donaldson I, Emberton M, Ehdaie B, Hadaschik BA, Marks LS et al (2015) Detection of clinically significant prostate cancer using magnetic resonance imaging-ultrasound fusion targeted biopsy: a systematic review. Eur Urol 68(1):8-19. doi:10.1016/j.eururo.2014.10.026

10. Baco E, Ukimura O, Rud E, Vlatkovic L, Svindland A, Aron M et al (2015) Magnetic resonance imaging-transrectal ultrasound image-fusion biopsies accurately characterize the index tumor: correlation with step-sectioned radical prostatectomy specimens in 135 patients. Eur Urol 67(4):787-794. doi:10.1016/j. eururo.2014.08.077 
11. Arsov C, Becker N, Rabenalt R, Hiester A, Quentin M, Dietzel $F$ et al (2015) The use of targeted MR-guided prostate biopsy reduces the risk of Gleason upgrading on radical prostatectomy. J Cancer Res Clin Oncol 141(11):2061-2068. doi:10.1007/ s00432-015-1991-5

12. Pokorny MR, De Rooij M, Duncan E, Schröder FH, Parkinson R, Barentsz JO et al (2014) Prospective study of diagnostic accuracy comparing prostate cancer detection by transrectal ultrasound-guided biopsy versus magnetic resonance (MR) imaging with subsequent MR-guided biopsy in men without previous prostate biopsies. Eur Urol 66(1):22-29

13. Wysock JS, Rosenkrantz AB, Huang WC, Stifelman MD, Lepor H, Deng FM et al (2014) A prospective, blinded comparison of magnetic resonance (MR) imaging-ultrasound fusion and visual estimation in the performance of MR-targeted prostate biopsy: the profus trial. Eur Urol 66(2):343-351. doi:10.1016/j. eururo.2013.10.048

14. Puech P, Rouvière O, Renard-Penna R (2013) Prostate cancer diagnosis: multiparametric MR-targeted biopsy with cognitive and transrectal US-MR fusion guidance versus systematic biopsy. Radiology 268(2):461-469

15. Haider MA, Yao X, Loblaw A, Finelli A (2014) Multiparametric magnetic resonance imaging in the diagnosis of prostate cancer: a systematic review. Clin Oncol [Internet]. Elsevier Ltd. Available from: http://linkinghub.elsevier.com/retrieve/pii/ S0936655516300991

16. Da Rosa MR, Milot L, Sugar L, Vesprini D, Chung H, Loblaw A et al (2015) A prospective comparison of MRI-US fused targeted biopsy versus systematic ultrasound-guided biopsy for detecting clinically significant prostate cancer in patients on active surveillance. J Magn Reson Imaging 41(1):220-225

17. Pepe P, Garufi A, Priolo G, Pennisi M (2015) Can MRI/TRUS fusion targeted biopsy replace saturation prostate biopsy in the re-evaluation of men in active surveillance? World J Urol. doi:10.1007/s00345-015-1749-3

18. Sonn GA, Chang E, Natarajan S, Margolis DJ, MacAiran M, Lieu P et al (2014) Value of targeted prostate biopsy using magnetic resonance-ultrasound fusion in men with prior negative biopsy and elevated prostate-specific antigen. Eur Urol 65(4):809-815. doi:10.1016/j.eururo.2013.03.025

19. Linstone HA, Turoff M (2002) The Delphi method-techniques and applications. Delphi Method-Tech Appl [Internet]. 1-616. Available from: http://www.millennium-project.org/ FRMv3_0/04-Delphi.pdf

20. Ahmed HU, El-Shater Bosaily A, Brown LC, Kaplan RS, Colaco-Moraes Y, Ward K et al (2016) The PROMIS study: a paired-cohort, blinded confirmatory study evaluating the accuracy of multi-parametric MRI and TRUS biopsy in men with an elevated PSA. ASCO Meet Abstr [Internet]. 34(15_suppl):5000. Available from: http://meeting.ascopubs.org/cgi/content/ abstract/34/15_suppl/5000
21. Rosenkrantz AB, Verma S, Choyke P, Eberhardt SC, Eggener SE, Gaitonde K et al (2016) Prostate MRI and MRI-targeted biopsy in patients with a prior negative biopsy: a consensus statement of the American Urological Association and the Society of Abdominal Radiology's Prostate Cancer Disease-Focused Panel. J Urol [Internet]. Elsevier Ltd. Epub ahead. Available from: http://linkinghub.elsevier.com/retrieve/pii/S0022534716306590

22. Tamada T, Sone T, Jo Y, Yamamoto A, Yamashita T, Egashira N et al (2008) Prostate cancer: relationships between postbiopsy hemorrhage and tumor detectability at MR diagnosis. Radiology [Internet]. 248(2):531-539. Available from: http://www.ncbi. nlm.nih.gov/pubmed/18539890

23. Feng T, Koopman S, Kim HL (2015) Impact of post prostate biopsy hemorrhage on multiparametric magnetic resonance imaging. Can J Urol 22:5

24. Mertan FV, Greer MD, Shih JH, George AK, Kongnyuy M, Muthigi A et al (2016) Prospective evaluation of the prostate imaging reporting and data system version 2 (PI-RADSv2) for prostate cancer detection. J Urol [Internet]. Elsevier Ltd 2. Available from: http://www.sciencedirect.com/science/article/pii/ S0022534716301999

25. Le Nobin J, Rosenkrantz AB, Villers A, Orczyk C, Deng FM, Melamed J et al (2015) Image guided focal therapy for magnetic resonance imaging visible prostate cancer: defining a 3-Dimensional treatment margin based on magnetic resonance imaging histology co-registration analysis. J Urol 194(2):364-370

26. Rouvière O, Girouin N, Glas L, Ben Cheikh A, Gelet A, MègeLechevallier F et al (2010) Prostate cancer transrectal HIFU ablation: detection of local recurrences using T2-weighted and dynamic contrast-enhanced MRI. Eur Radiol 20(1):48-55

27. Shah TT, Kasivisvanathan V, Jameson C, Freeman A, Emberton M, Ahmed HU (2015) Histological outcomes after focal highintensity focused ultrasound and cryotherapy. World J Urol. doi:10.1007/s00345-015-1561-0

28. Mottet N, Bellmunt J, Briers E, van den Bergh RCN, Bolla M, van Casteren NJ et al (2015) Guidelines on prostate cancer. Eur Assoc Urol Guidel [Internet] 1-156. Available from: www. uroweb.org/gls/pockets/english/07_Prostate_Cancer.pdf

29. Donaldson IA, Alonzi R, Barratt D, Barret E, Berge V, Bott S et al (2014) Focal therapy: patients, interventions, and outcomes - a report from a consensus meeting. Eur Urol [Internet]. European Association of Urology 67-73. Available from: http:// www.ncbi.nlm.nih.gov/pubmed/25281389

30. Muller BG, van den Bos W, Brausi M, Fütterer JJ, Ghai S, Pinto PA et al (2015) Follow-up modalities in focal therapy for prostate cancer: results from a Delphi consensus project. World J Urol [Internet]. Available from: http://www.ncbi.nlm.nih.gov/ pubmed/25559111 